\title{
Correction to: Preconditioning exercise reduces brain damage and neuronal apoptosis through enhanced endogenous 14-3-3 $\gamma$ after focal brain ischemia in rats
}

\author{
Shotaro Otsuka ${ }^{1} \cdot$ Harutoshi Sakakima ${ }^{1} \cdot$ Takuto Terashi ${ }^{1}$ Seiya Takada ${ }^{1} \cdot$ Kazuki Nakanishi $^{1} \cdot$ Kiyoshi Kikuchi $^{2,3,4,5}$
}

Published online: 17 December 2018

(c) Springer-Verlag GmbH Germany, part of Springer Nature 2018

\section{Correction to: Brain Structure and Function https://doi.org/10.1007/s00429-018-1800-4}

In the original publication of the article, both the Figs. $2 \mathrm{e}$ and $7 \mathrm{e}$ have been published incorrectly and the correct Figs. 2 and 7 are given below. The original article has been corrected.

The original article can be found online at https://doi.org/10.1007/ s00429-018-1800-4.

Harutoshi Sakakima

sakaki@health.nop.kagoshima-u.ac.jp

$\triangle$ Kiyoshi Kikuchi

kikuchi_kiyoshi@kurume-u.ac.jp

1 Course of Physical Therapy, School of Health Sciences, Faculty of Medicine, Kagoshima University, 8-35-1,

Sakuragaoka, Kagoshima 890-8544, Japan

2 Division of Brain Science, Department of Physiology, Kurume University School of Medicine, Kurume, Japan

3 Department of Neurosurgery, Kurume University School of Medicine, Kurume, Japan

4 Department of Systems Biology in Thromboregulation, Kagoshima University Graduate School of Medical and Dental Science, Kagoshima, Japan

5 Department of Pharmacology, Faculty of Dentistry, Mahidol University, Bangkok, Thailand 
Fig. 2 Preconditioning exercise enhanced the expression of 14-3-3 $\gamma$ in neurons and astrocytes. 14-3-3 $\gamma$ immunoreactivity surrounding the lesion significantly increased in the Ex-only group than in the control group (a, $\mathbf{b}, n=6)$. Representative western blotting and semi-quantitative analysis showed that protein levels of $14-3-3 \gamma$ significantly increased in the Ex-only group than in the control group (c, $\mathbf{d}, n=5$ and 4 , respectively). The 14-3-3 $\gamma$ positive cells were colocalized with NeuN or GFAP positive cells (e). Date are presented as mean \pm SE. $* p<0.05$. Scale bar $=50 \mu \mathrm{m}(\mathbf{a})$ or $20 \mu \mathrm{m}(\mathbf{e})$
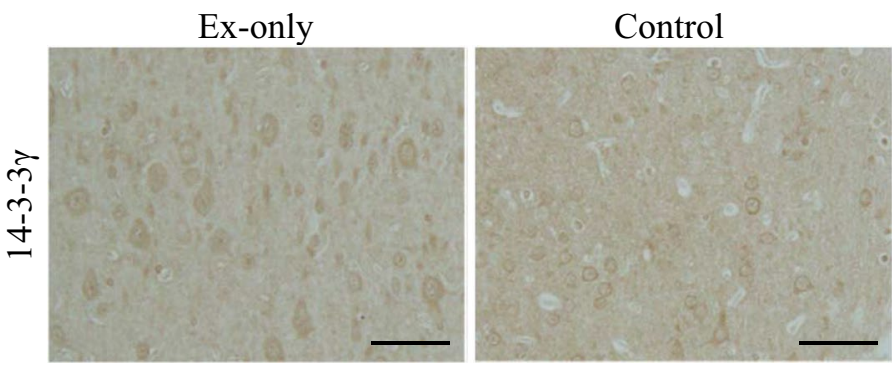

a
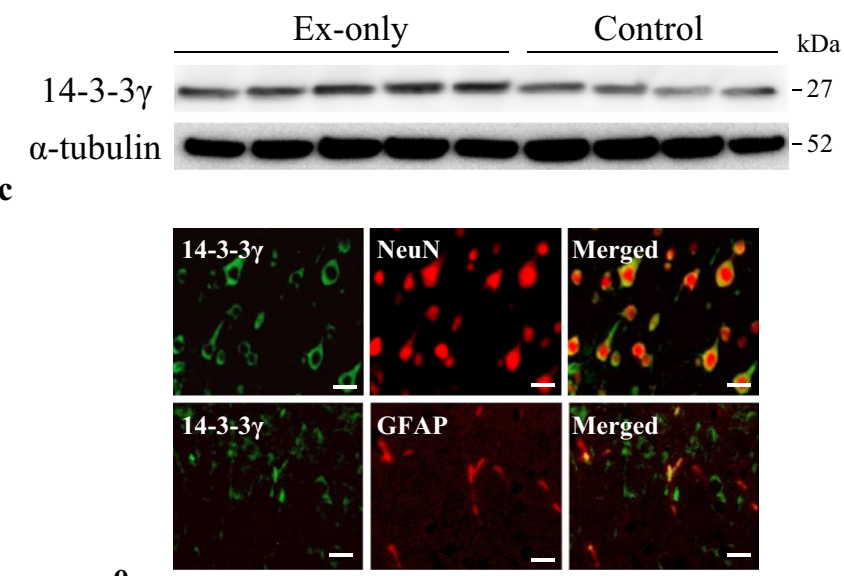
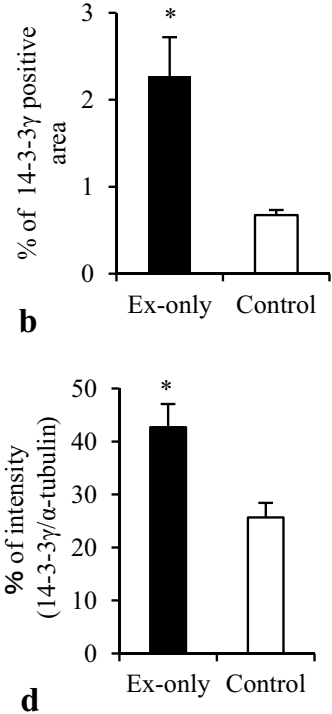

d
Fig. 7 The expression of caspase 3 and Bax surrounding the lesion after MCAO. The left side of each photomicrograph (a) shows an ischemic region, and the right side shows a surrounding the lesion (penumbral area). The caspase 3 and Bax immunoreactivities surrounding the lesion were significantly reduced in the Ex group $(\mathbf{a}-\mathbf{c})$. The caspase 3 immunopositive cells were co-localized with the neuronal marker (NeuN) and astrocyte marker (GFAP) in the Ex group (d), largely observed with the neuronal marker surrounding the lesion. The ratios of survival neurons (arrow) were significantly increased in the Ex group compared to that in the No-Ex group (e). Date are presented as mean $\pm \operatorname{SE}(n=7)$. ${ }^{*} p<0.05$. Scale bar $=50 \mu \mathrm{m}(\mathbf{a})$ or $20 \mu \mathrm{m}(\mathbf{d})$
Ex

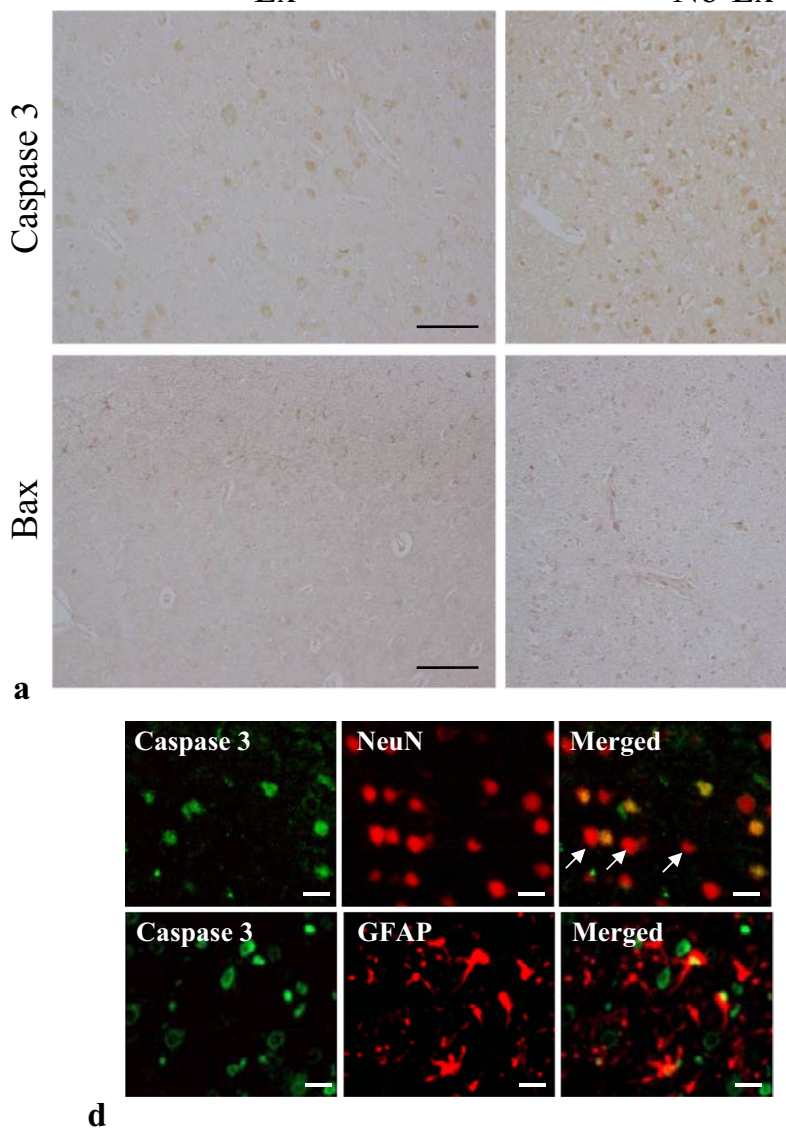

No-Ex
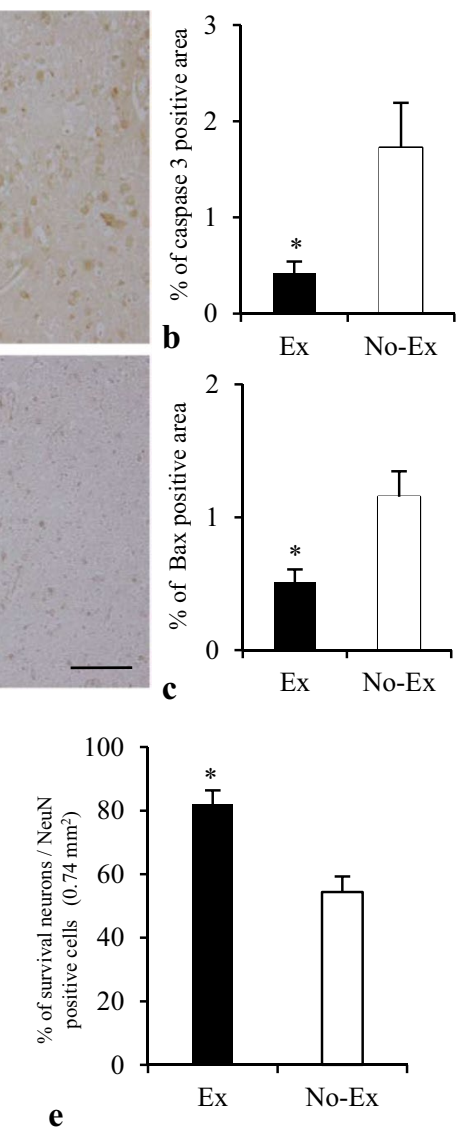